\title{
A remote test method for power grid security and stability control system and its engineering application
}

\author{
Qi Guo ${ }^{1,2,3}$, Yihua Zhu ${ }^{1,2,3}$, Dongxu Chang ${ }^{1,2,3}$, Shengnan $\mathrm{Li}^{4}$, Yong Chen ${ }^{4}$, and Mingkang \\ $\mathrm{Wu}^{1,2,3, *}$ \\ ${ }^{1}$ State Key Laboratory of HVDC, Electric Power Research Institute, China Southern Power Grid, \\ 510663 Guangzhou, China \\ ${ }^{2}$ Guangdong Provincial Key Laboratory of Intelligent Operation and Control for New Energy Power \\ System, 510663 Guangzhou, China \\ ${ }^{3}$ CSG Key Laboratory for Power System Simulation, Electric Power Research Institute, China \\ Southern Power Grid, 510663 Guangzhou, China \\ ${ }^{4}$ Electric Power Research Institute, Ltd. of Yunnan Power Grid Co., 650200 Kunming, China
}

\begin{abstract}
Security and stability control (SSC) system or special protection system (SPS) is an important means to ensure the safe operation of power grid. In view of the insufficiency that the current open-loop testing method cannot fully test the SSC system on-site, a remote real-time closed-loop test method is proposed. The solutions of key techniques such as information interaction, remote communication and signal conversion in remote test system are studied. Then the platform architecture and standard interface of the remote test system are designed. A remote test platform for SSC system is developed, and there application modes of the platform is deeply analysed. Finally, taking SSC system of Yunnan AC power outgoing grid as pilot application, experiment of two substations on-site is carried out. The results in practical project further prove the effectiveness of the method presented in this paper.

Keywords: Security and stability control system; Real-time simulation; Power communication network; Remote closed loop test.
\end{abstract}

\section{Introduction}

With the construction of UHV(Ultra-High Voltage) power grid and the large application of new energy power, HVDC(High Voltage Direct Current Transmission) and power electronic devices[1], the scale and complexity of power system are increasing day by day, which brings more and more severe challenges to the security and stability of power system[2]. As the second line of defence to ensure the security and stability of power system, Security and Stability Control system(SSC) is widely used in the power grid and has become an indispensable part of the power grid[3].

The complexity of large-scale SSC has brought great challenges to the test work due to

\footnotetext{
*Corresponding author: wumk@csg.cn
} 
its wide distribution area, involving many plants and stations, and various types of devices[4]. As open-loop test, the test environment of the traditional method has a large gap with the real power grid[5], which cannot actually investigate the operation characteristics of the SSC strategy after the fault and the response characteristics of the power grid. In view of the above shortcomings[6], the reference [7-8] proposed a test system based on real-time digital simulation technology in closed-loop, and then carried out field debugging. However, on the one hand, due to the limitation of lab environment and long distance transportation, the centralized testing method in laboratory cannot test all the stations, which is difficult to reveal the hidden problems of on-site SSC[9]. On the other hand, the simulation of complex faults cannot be completed at the SSC spot, and the low automation requires a lot of manpower and material resources.

In view of the above shortcomings, this paper proposes a remote test method for power grid security and stability control system, which uses the field SSC and the existing electric power communication network, carries out large-scale joint simulation test of the field SSC in the laboratory environment. The feasibility and availability of the remote test method has been proved via carrying out the test of an existing field SSC by real time simulation.

\section{The remote test method of SSC}

The power system has formed a special power communication network architecture with optical fiber communication as the main feature, hierarchical and self-healing ring network as the main characteristics[10-12]. The high-speed and large-capacity communication ability provides the technical basis for the remote test method of SSC[13]. Generally, when checking the stability characteristics of power system, the time from the beginning of fault to the completion of the SSC control measures is $300 \mathrm{~ms}$, which should also be chosen as the index of the remote test method of SSC[14].

\subsection{Information exchange between laboratory and field}

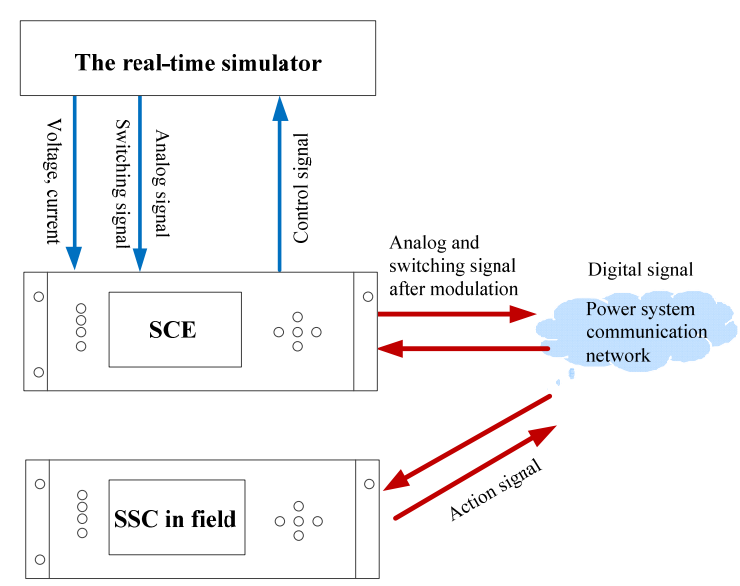

Fig. 1. Information flow of SSC remote test platform.

The conventional real-time simulation test is a centralized test. In the laboratory environment, the real-time simulation system directly interacts with the tested SSC for analog and switching signal by GTIO, identically, in the remote test scenario, the information interaction is completed through the power communication network based on the new interaction method. Therefore, Signal Conversion Equipment(SCE) is produced to complete the real-time communication task of remote test. As the core equipment of remote 
SSC test, the device is the link between the real-time simulation system and the field SSC. It mainly completes the following two core logics:

- The analog and switch signals from the real-time simulation system are converted into digital signals that can be identified by the field SSC. The signals are transmitted to the wide area distributed SSC through the power system communication network. Instead of the field signals, The signals from the SCE are used as the input for the SSC.

- The action signal from the field SSC is fed back to the SCE through the power system communication network. After the SCE identification and conversion, the signal is fed back to the real-time simulation system, which works in closed-loop control.

In the remote test mode, the information flow between the real-time simulation system in laboratory and the field SSC is shown in figure 1.

\subsection{The group time of the remote SSC test}

In the remote test mode of SSC, the group time refers to the time from the beginning of grid fault in simulation system, fault detection of SSC, to the execution of the control order in simulation system.

A typical SSC including three levels of control master station, control sub-station and executive station is taken as an example in Figure 2, which shows the group time including $\Delta \mathrm{t}_{\text {cal }}, \Delta \mathrm{t}_{\text {up }}, \Delta \mathrm{t}_{\text {ctrl }}, \Delta \mathrm{t}_{\text {down }}, \Delta \mathrm{t}_{\text {ope }}$ in the remote test mode.

- $\Delta \mathrm{t}_{\mathrm{cal}}$ : Time from triggering fault of simulation system to detection of fault by signal conversion device. According to FFT algorithm, the process of data sampling needs a periodic data column, the typical value is $20 \mathrm{~ms}$.

- $\Delta \mathrm{t}_{\mathrm{up}}$ : Time from the SCE detecting fault to starting of tested SSC. Including internal calculation and communication delay of SCE, communication delay between SCE and SSC, and command confirmation time of SCE.

- $\Delta \mathrm{t}_{\text {ctrl }}$ : The time from the start of the first level SSC to the time when the control command of the last level SSC is issued (excluding the relay action time), including the time from the start of the first level SSC to the time when the relay receives the control signal $\left(\mathrm{t}_{\text {brk }}\right)$, the SSC executing time $\left(\mathrm{t}_{\mathrm{set}}\right)$ excluding action time of the relay of last level $\operatorname{SSC}\left(t_{\text {relay }}\right)$.

- $\Delta \mathfrak{t}_{\text {down }}$ : The time when the last level SSC control command is sent to the SCE, including the communication delay between the last level SSC and the SCE, the command confirmation time of the SCE, and the internal calculation and communication delay of the SCE.

- $\Delta \mathrm{t}_{\mathrm{ope}}$ : The time from the last level SSC control command is sent to the simulator to the time when the simulator completes the control measures, including the operation time of the action relay(5ms typically), the communication delay between the last level SSC and the simulation system $(0.05 \sim 0.1 \mathrm{~ms})$, and the breaker action time( $40 \mathrm{~ms}$ typically).

Compared with the centralized simulation test in the laboratory, the remote test increases two delays, $\Delta \mathfrak{t}_{\text {up }}$ and $\Delta \mathfrak{t}_{\text {down. }}$. According to the measured data of ASON network transmitted by the backbone of China Southern Power Grid(CSG), the one-way delay of $2 \mathrm{M}$ service communication link of the SSC is generally no more than $10 \mathrm{~ms}$. At the same time, by optimizing the data conversion efficiency of the SCE, the internal calculation and communication time of the SCE can be controlled to be less than $3 \mathrm{~ms}$, the confirmation command time of SSC is fixed as 3-frame message confirmation time $(5 \mathrm{~ms})$. Therefore, it can be estimated that the critical delay of the whole group of closed-loop time of remote test is less than $36 \mathrm{~ms}$.

Taking $500 \mathrm{kV}$ AC line fault trip as an example, combined with the analysis of long time fault-free tripping criterion, the time $t_{\text {brk }}$ is generally less than $100 \mathrm{~ms}$. In addition, according 
to the requirements of general technical conditions, the group action time of SSC should be controlled within $100 \mathrm{~ms}$. So, the time $\left(\Delta \mathrm{t}_{\text {ctrl }}\right)$ refers to:

$$
\begin{aligned}
\Delta t_{c r r l}=t_{\text {brk }}+t_{\text {set }}-t_{\text {relay }} \leq 100+100-t_{\text {relay }} \\
\\
\Delta t_{\text {cal }}+\Delta t_{\text {crrl }}+\Delta t_{\text {ope }} \\
\leq 20+200-t_{\text {relay }}+t_{\text {relay }}+40 \\
=260 \mathrm{~ms}
\end{aligned}
$$

So, the group time of remote closed loop test is:

$$
t_{\text {loop }}=\Delta t_{\text {cal }}+\Delta t_{\text {up }}+\Delta t_{c t r l}+\Delta t_{\text {down }}+\Delta t_{\text {ope }} \leq 296 m s
$$

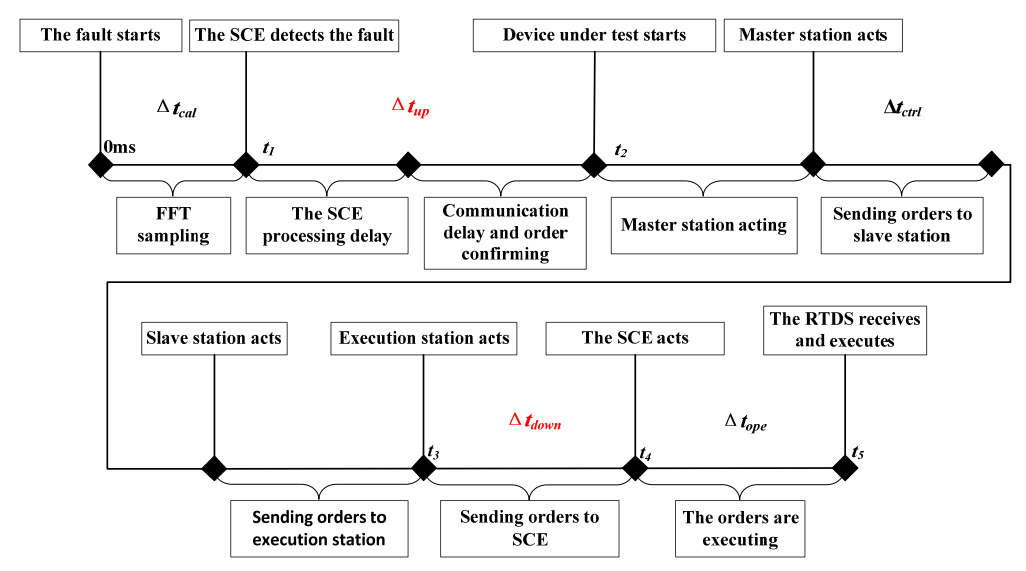

Fig. 2. Timing sequence of remote test.

In engineering, the group action time of SSC is generally less than $100 \mathrm{~ms}$, especially DC blocking criterion and line fault tripping criterion relying on relay protection action signal, which can reduce tens of milliseconds. Therefore, the group time of remote closedloop test can be controlled within $300 \mathrm{~ms}$ to meet the control time requirements of system analysis and test.

\subsection{Remote closed loop test communication technology}

The high level data link control (HDLC) point-to-point communication is adopted in the communication between the SSC. The application layer data is completely transparent and user-defined, and the transmission data volume is relatively small, and the bandwidth margin is large. Based on HDLC, the inter station-to-station communication scheme of the remote test system of SSC can be designed:

- Communication channel: the laboratory is equipped with synchronous digital hierarchy equipment (SDH) which meets the communication management and safety design standards of SSC, and is connected to the main communication network of power system. The point-to-point communication is adopted between the interface conversion device and the SSC of the tested plants and stations, and a $2 \mathrm{Mbps}$ communication dedicated or multiplex channel is adopted; an E1 interface conforming to the G.703 standard is added to each tested station to open the $2 \mathrm{Mbps}$ service channel to the laboratory.

- Communication protocol: HDLC protocol is adopted between the signal conversion device and the stability device of each tested station. The coding mode is consistent with 
the communication coding mode between the stable device stations. The information coding layer and baseband transmission coding layer adopt 1b4b and HDB3 coding respectively to transmit the analog quantity, switching value and action information collected by the tested device.

- Transmission rate: compatible with the channel receiving and transmitting rate of the existing SSC, the data (command, device status) with high real-time requirements is designed as 600 frames/s; the data (analog and switching signals) with relatively low realtime requirements are designed with time-sharing multiplexing, which can be designed as $150 \mathrm{frames} / \mathrm{s}$, and the frame protocol adopts strict mechanism to ensure the reliability of the data transmission; the communication frame length is 28bytes, which can ensure that network storm will not occur and affect other services of transmission network.

\section{Architecture and interface design of remote test platform}

\subsection{The architecture of remote test platform}

According to the above methods, a remote test platform for SSC is designed based on RTDS real-time digital simulation system. The overall architecture is shown in Figure 3. The test platform is composed of RTDS simulator, signal conversion device, communication interface device, tested SSC and monitoring system, and realizes communication by relying on power communication network. Laboratory simulation system and field wide area distributed SSC constitute a closed-loop test system.

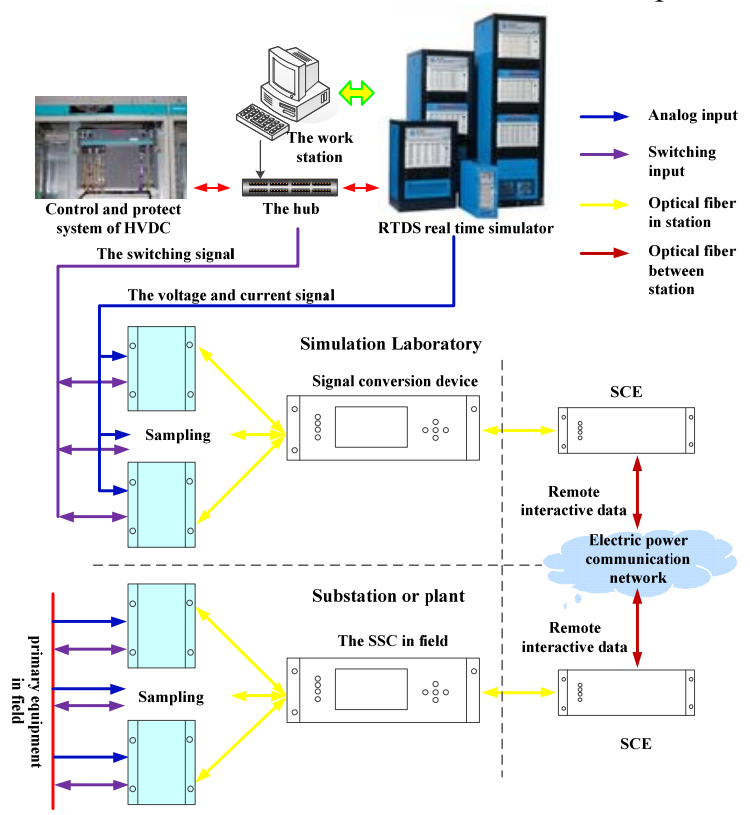

Fig. 3. Architecture of SSC remote test platform.

- Parameter configuration: In order to realize the one-to-one correspondence of the voltage, current, calculated power, operation and fault status, as well as the input and output quantity collected by the SCE and the tested equipment, a set of configuration parameters is designed for each station to be tested in the signal conversion device. According to the actual signal collected by the station, it can be directly configured by using the fixed value, and the quantity can be sent to the corresponding station. In addition, in order to adapt to 
the strategy application that needs to distinguish the type of fault trip, the A-phase trip, Bphase trip, c-phase trip and HWJ signals are configured for each interval. The device can access the corresponding signals and their combination according to the needs. According to the setting value, the fault type of corresponding interval (the same as the corresponding analog configuration interval) is selected and sent to the corresponding station.

- Key control parameters: For the sake of safety, the remote test platform is controlled to ensure the normal operation of the on-site stability device. The control word "test station $\mathrm{x}$ remote test valid" is set in SCE: which means the test station $\mathrm{x}$ enters the remote test mode, receives the data provided by the SCE for strategy calculation and logic output. When the control word is invalid, the device operates normally. At the same time, in order to study the accuracy and reliability of the SSC in the laboratory environment considering the transmission delay, the SCE sets the "test station channel delay time" parameter, and the fixed channel delay simulation is realized by software between the signal conversion device and the test station.

\subsection{The Interface between simulation system and SCE}

The simulation system provides analog signals and corresponding HWJ switching signals to the SCE through the interface card, and obtains the power, on-off, start-up and trip information of sudden variables by the slave device collection, calculation and judgment; the DC operation status information is sent to the SCE by the DC control and protection system in the form of hard contact or optical fiber. At the same time, the SCE receives the information of load shedding or DC modulation from the field device and sends it to the simulation system or DC control and protection system, so as to realize the one-to-one correspondence between the analog and the switching value of the simulation system through the signal conversion device and the field device.

\subsection{The interface between SCE and the field SSC}

In order to increase the maintainability and reusability of the system, the SCE is standardized. According to the AC and DC information provided by the RTDS system, and combined with the current device communication common protocol, the data is comprehensively planned. The communication interface is shown in Table 1.

Table 1. Communication content between SCE and SSC equipment on-site.

\begin{tabular}{|c|c|}
\hline SCE to SSC & SSC to SCE \\
\hline Frame header & Frame header \\
\hline Operation information of AC interval & AC command 1 \\
\hline Burst start information of AC interval & AC command 2 \\
\hline Trip information of AC interval & DC command 1 \\
\hline Operation and fault information of pole1 & DC command 2 \\
\hline Operation and fault information of pole1 & Reserve \\
\hline Power serial number(0-2) & Reserve \\
\hline Power 1 & Reserve \\
\hline Power 2 & Reserve \\
\hline Power 3 & Reserve \\
\hline Power 4 & Reserve \\
\hline
\end{tabular}

\subsection{Design of remote interface for the field SSC}

The communication channel between the field SSC and the SCE should be added, and the 
software needs to be modified on the original basis. In order to effectively distinguish the data collected from the actual power grid and the data obtained from the SCE, a control word of "remote test function input" should be added to the software of the stability device. Only when the control word and the "test switch" of the SSC device are put into operation at the same time, the data of the SCE is valid.

The software of SSC needs to be modified to complete the following functions:

- Receive the analog and switch information provided by the simulation system, replace the actual analog and switch value collected by the field v-i transformer, and fully correspond to the actual value.

- When the test module is put into operation and the equipment is triggered by simulation data, the control signals of test device is fed back to the SCE. If it is necessary to synchronously test the performance of on-site trip circuit, the action signals can also be driven to the circuit breaker or other mechanical mechanisms to complete the trip test.

\section{The trial in the field}

\subsection{Overall scheme}

The SSC of Yunnan AC transmission section for power transmission from west to east of CSG is used for field application, which includes Luoping station, Yanshan Station, Baofeng station, Manwan Power Plant and Dachaoshan Power Plant. Among them, the field devices of Luoping station and Yanshan Station are connected to the remote test platform, other stations are connected to the platform with laboratory simulation device. The optical transmission equipment of the laboratory is connected to the trunk transmission ASON network of CSG and connected to the $2 \mathrm{Mbps}$ communication channel of Luoping station and Yanshan Station, thus forming a wide area closed-loop control system. The topology of the test system is shown in Figure 4.

\subsection{The union debugging in the field}

First of all, DDF ring is established in Luoping station and Yanshan Station through optical transmission equipment. Then, the laboratory communication interface device sends and receives information from the field device to test the communication delay between the laboratory and the site. The measured data are shown in Table 2. It can be seen that the communication delay has a larger margin than the artificially set delay in the laboratory.

Table 2. Communication latency of links between stations.

\begin{tabular}{|c|c|c|}
\hline Communication link & Self loop point & $\begin{array}{c}\text { Bidirectional } \\
\text { communication delay(ms) }\end{array}$ \\
\hline Laboratory to Luoping station & Luoping DDF rack & 10.00 \\
\hline Laboratory to Yanshan station & Yanshan DDF rack & 9.17 \\
\hline
\end{tabular}

On this basis, according to the topology shown in Figure 4, the field SSC is connected to the remote test system for union debugging. Taking Luo-Bai double line N-2 fault under the overhaul mode of Luo-Ma line as an example, according to the preset control strategy of SSC, when the power of Luoping $500 \mathrm{kV}$ AC transmission section before the accident is greater than Yunnan cut-off threshold value, once Luo-Bai double line trip occurs, the SSC will detect the fault and send the cut-off quantity to Yunnan Baofeng station. The SSC of Baofeng station will calculate the cut-off sequence according to the cut-out quantity, and then send the cut-off command to Manwan Power Plant and Dachaoshan Power Plant 
respectively.

The group time of remote closed-loop test is shown in Figure 5.

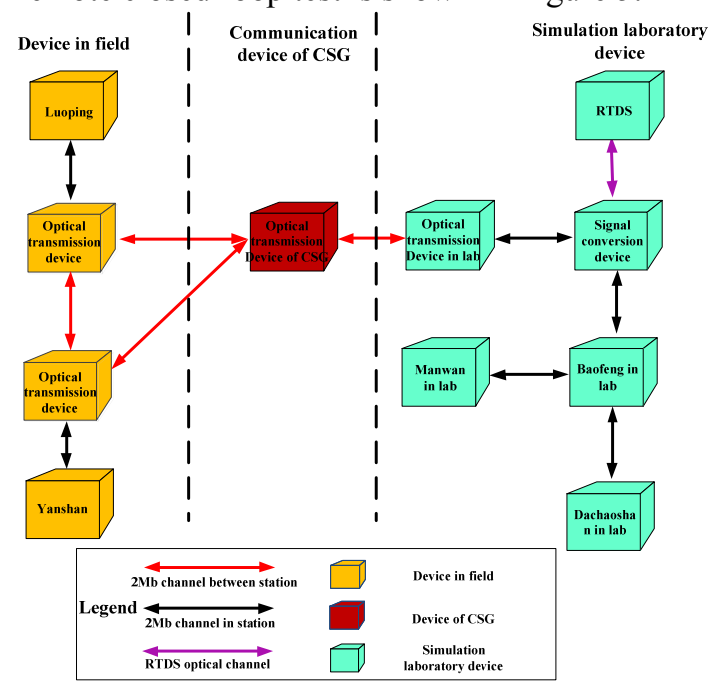

Fig. 4. Topology diagram of remote test system of trial application.

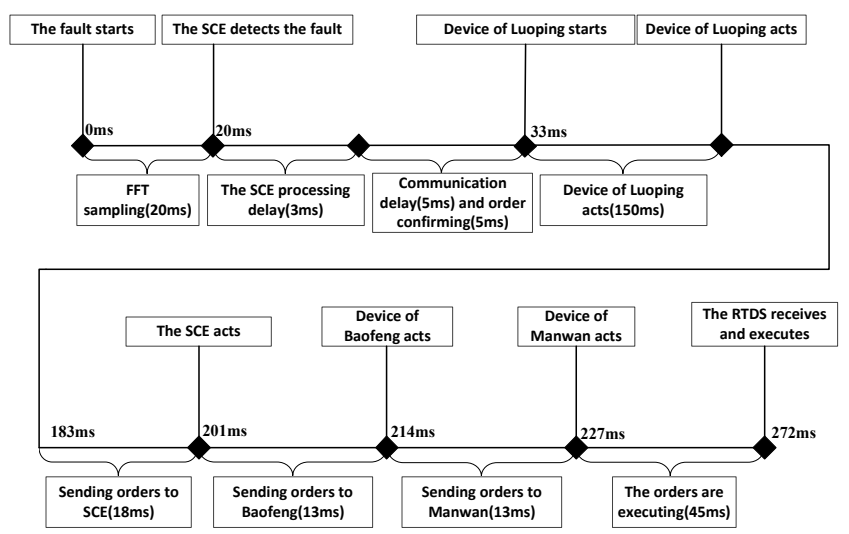

Fig. 5. Timing sequence of remote test on-site.

- The transmission line Luo-Bai I suffered three-phase short-circuit fault at 0s, then, Luo-Bai I and Luo-Bai II were cut-off from the power system at $0.1 \mathrm{~s}$. The SSC device in Luoping Station detected the event at the time $0.188 \mathrm{~s}$ and sent out control measures to cut off the essential $653 \mathrm{MW}$ power from the power system after the calculation of the SSC device.

- Then, the SCE received the order from the SSC device of Luoping at 0.206s and the SSC device of Baofeng received the order at $0.217 \mathrm{~s}$. After the calculation of the SSC device of Baofeng, it sent out orders to cut off the \#1 generator and the \#2 generator of Manwan Power Plant, and the \#1 generator and the \#3 generator of Dachaosha Power Plant. The SSC device of Manwan and Dachaoshan received cut-off order at $0.227 \mathrm{~s}$.

- At the time 0.272s, the RTDS received cut-off orders and implemented the orders. Finally, the power system returned to stable operation after the fault, which proved the effectiveness of SSC control measure.

According to RTDS recording, the group time of remote closed-loop test is about $17 \mathrm{~ms}$ longer than that of conventional test, which is basically consistent with the $26 \mathrm{~ms}$ by 
theoretical analysis. Under the two test modes, the operation characteristics of the SSC are consistent, the power grid is stable, and the dynamic response characteristics of Yunnan transmission lines are consistent after the fault, as shown in Figure 6.

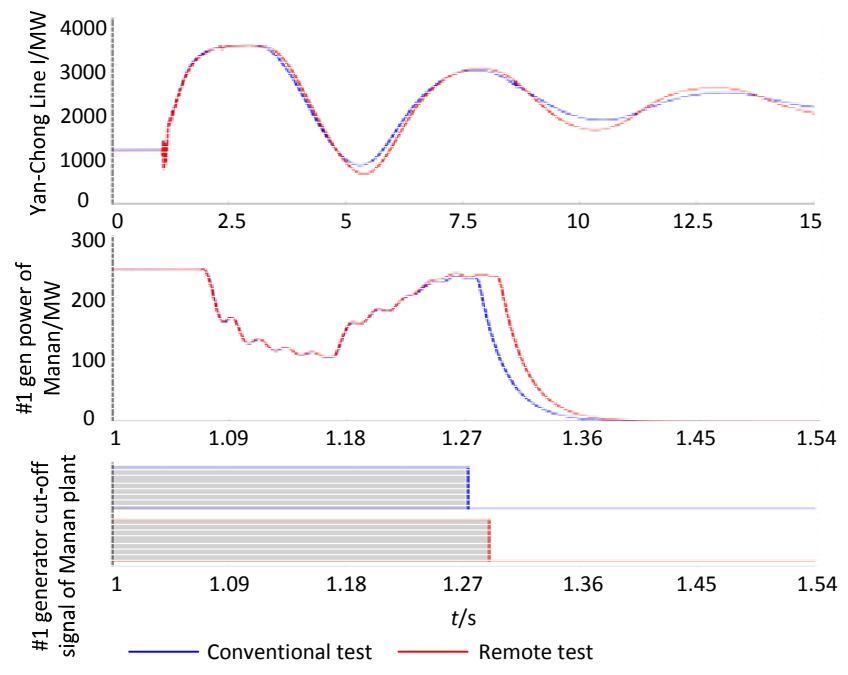

Fig. 6. Contrast of on-site conventional test and remote test.

After the detailed analysis of the action sequence of each link of the remote test system, and compared with the conventional test, the conclusion is as follows:

- The SSC devices tested in field are not affected by the communication delay. When the communication is normal, they can correctly identify the analog and switch signal sent from the SCE, and act correctly according to the strategy table of SSC devices.

- The group time of remote closed-loop test only increases the communication link delay and device processing delay, and will not affect the action results of the SSC.

According to the measured data of communication delay, the group time of remote closed-loop test can still meet the requirement of less than $300 \mathrm{~ms}$. The power system in simulation system can maintain stable operation after the strategy action, and the test results of field SSC are consistent with the conventional RTDS simulation test results.

\section{Conclusion}

This paper presents a remote closed-loop test method of SSC based on real-time simulation system. The feasibility and effectiveness of the technical route in practical projects are successfully verified through the field union debugging in Yunnan AC SSC of CSG at the cost of adding communication and test modules to the program of the device in the field. The SSC remote test platform developed in this paper can effectively solve the difficulty of dynamic closed-loop test for wide area distributed SSC in field, realize standardized and efficient wide area closed-loop test, and provide a new technical direction for control and protection system testing in field. In the future, it will be a research direction to decouple the remote test method from the field SSC.

\section{Acknowledgement}

Project Supported by Science and Technology Project of China Southern Power Grid Company Limited. (ZBKJXM20190039). 


\section{References}

1. Yunhao Peng, Xijian Dong, Haiqiang Zhou, et al. Reliability evaluation of power grid security and stability control system $[\mathrm{J}]$. Power System Protection and Control, 2020,48(13):123-131.

2. Dongxu Chang, Qi Guo, Yihua Zhu, et al. Interface Design and Simulation of Multiterminal Hybrid HVDC and Security and Stability Control System [J]. Power System Technology, 1-9[2021-04-02].https://doi.org/10.13335/j.1000-3673.pst.2020.1695.

3. LIANG Shaohua, XIA Shangxue, HUO Chao, et al. Interface and Coordination Control Method Between Qinghai-Tibet DC Stability Control System and Pole Control System[J]. Automation of Electric Power Systems, 2014, 38(8): 125-128.

4. Lili ZHAO, Xueming LI, Ming NI, et al. Review and prospect of hidden failure: protection system and security and stability control system[J]. Journal of Modern Power Systems and Clean Energy, 2019, 7(6): 1735-1743.

5. WANG Liang, WANG Xinbao, GAO Liang, et al. A Fault Scenario Based Method for the Regional Power Grid Security and Stability Control System Test[J]. Automation of Electric Power Systems, 2007, 31(18): 39-42.

6. LI Xingjian, XIA Yanhui, CHEN Songlin, et al. Development and application of distributed simulation \& test system for stability control system[J]. Electric Power Automation Equipment, 2014, 34(5): 163-168.

7. GUO Qi, HAN Weiqiang, ZENG Yonggang, et al. Security and Stability Control Technology and Research Platform Based on Real-time Simulation Part One Framework and Characteristics[J]. Automation of Electric Power Systems, 2012, 36(20): 1-5.

8. GUO Qi, HAN Weiqiang, XU Guanghu, et al. Security and Stability Control Technology Test and Research Platform Based on Real-time Simulation Part Two Application Examples[J]. Automation of Electric Power Systems, 2012, 36(21): 19-23.

9. Zhao Lili, LI Xueming, NI Ming, et al. Review and Prospect of Research on Hidden Failures of Protection System and Security and Stability Control System[J]. Automation of Electric Power Systems, 2014, 38(22): 128-133.

10. ZHOU Hualiang, ZHENG Yuping, JIANG Lei, et al. A Distributed Real-time Control Technology Based on Point-to-point Communication[J]. Automation of Electric Power Systems, 2015, 39(10): 107-111.

11. JING Shi, HUANG Qi, ZHEN Wei, et al. A Panoramic Test System for Intelligent Substation Based on Wireless Time Synchronization Technology[J]. Automation of Electric Power Systems, 2013, 37(2): 72-78.

12. LI Juan, JIAO Shaohua, XIAO Shiwu. A Multi-terminal and Closed-loop Power System Real-time Testing Simulator at Different Substations[J]. Automation of Electric Power Systems, 2007, 31(10): 97-101.

13. General technical conditions of power system security and stability control system: DL/T 1092-2008[S]. Beijing: Standards Press of China, 2008.

14. LI Juan, JIAO Shaohua, XIAO Shiwu. A Multi-terminal and Closed-loop Power System Real-time Testing Simulator at Different Substations[J]. Automation of Electric Power Systems, 2007, 31(10): 97-101. 